\title{
Projeção de distribuição diamétrica de povoamento comercial de eucalipto utilizando área basal
}

\author{
Projection of diametric distribution of Eucalyptus stands using basal area
}

\author{
Lidiomar Soares da Costa ${ }^{1 *}$, Renato Vinícius Oliveira Castro², \\ Ana Flávia Neves Mendes Castro², Leonidas Soares Murta Júnior ${ }^{3}$, \\ Erica Karolina Barros de Oliveira ${ }^{1}$ e Ilvan Medeiros Lustosa Junior ${ }^{1}$
}

\begin{abstract}
Resumo
O objetivo do presente trabalho foi projetar a distribuição diamétrica com inclusão da variável área basal no sistema de equações e avaliar a eficiência desse modelo na projeção da distribuição diamétrica de plantios comerciais do híbrido de Eucalyptus urophylla x Eucalyptus grandis. Os dados foram obtidos de inventários florestais em parcelas permanentes de área média de 291,5 m², em idades variando de 19 a 98 meses em povoamento comercial. Ajustou-se a função densidade de probabilidade Weibull de dois parâmetros pelo método da máxima verossimilhança e, para a projeção dos parâmetros dessa função utilizou-se um sistema de equações com inclusão da variável área basal. A avaliação do ajuste desse sistema foi feita por meio da análise gráfica de resíduos e das estatísticas de precisão, coeficiente de determinação ajustado e erro padrão da média relativo, além da comparação entre valores estimados e observados por meio do teste de Kolmogorov-Smirnov. A função Weibull foi aderente para $98 \%$ dos ajustes e as estimativas das variáveis do povoamento e parâmetros da função Weibull apresentaram $R_{\text {ajustado }}^{2}$ alto, erro padrão baixo e baixa dispersão de resíduos. As distribuições diamétricas estimadas foram consistentes do ponto de vista do realismo biológico e mais precisas com a utilização da área basal no sistema de equações. A distribuição diamétrica de povoamento comercial de eucalipto projetada com a inclusão de área basal no sistema de equações para projeção dos parâmetros da função Weibull apresenta consistência, além de ser eficiente para, pelo menos, quatro prognoses consecutivas.
\end{abstract}

Palavras-chave: Manejo florestal, prognose florestal, classe diamétrica

\begin{abstract}
his study aimed to project the diametric distribution with inclusion of the basal area in the system of equations and evaluate the efficiency of this model in the projection of the diametric distribution of commercial plantations of the hybrid of Eucalyptus urophylla x Eucalyptus grandis. The data were obtained by forest inventories in permanent plots with a mean area of $291.5 \mathrm{~m}^{2}$, ranging from 19 to 98 months in commercial stands. The Weibull probability density function was adjusted by the maximum likelihood method and, for the projection of the parameter of that function, a system of equations with the inclusion of the variable basal area. The evaluation of the adjustment of this system was performed by the graphical analysis of residues and precision statistics like adjusted coefficient of determination and standard error of the relative mean, in addition to the comparison between estimated and observed values using the Kolmogorov-Smirnov test. The Weibull function was adherent to $98 \%$ of the adjustments and the estimates of the stands variables and parameters of the Weibull function showed high adjusted coefficient of determination, low standard error and low residue dispersion. The estimated diametric distributions were consistent from the point of view of biological realism and more accurate with the use of the basal area in the system of equations. The estimated diameter distribution of eucalyptus stands, with incluision of basal area in equations system for projection Weibull function parameters, presents consistency, in addition it is efficient for at least four consecutive prognoses.
\end{abstract}

Keywords: Forest management, forest prognosis, diametric class

1. Departamento de Engenharia Florestal, Universidade de Brasília - UnB. Brasília / DF, Brasil. * Autor Correspondente: lidiomar.ef@gmail.com

2. Departamento de Ciências Agrárias, Universidade Federal de São João Del-Rei - UFSJ. São João Del-Rei / MG, Brasil.

3. Instituto Estadual de Floresta - IEF. Teófilo Otoni / MG, Brasil.

Sci. For., Piracicaba, v. 47, n. 122, p. 265-272, jun. 2019

DOI: doi.org/10.18671/scifor.v47n122.09 


\section{INTRODUÇÃO}

O planejamento é parte fundamental do processo de produção visando a obtenção de sucesso, e para isso tem-se à disposição diversas ferramentas e estratégias. No setor florestal, uma dessas ferramentas é a modelagem do crescimento e produção em nível de distribuição diamétrica que tem sido amplamente utilizada e permite o conhecimento do estágio de desenvolvimento do povoamento, além da previsão e simulação da produção futura de madeira (SIIPILEHTO et al., 2007). Com isso, contribui para o sucesso de diversas atividades como a colheita e sortimento de produtos florestais (BURKHART; TOMÉ, 2012).

A modelagem da distribuição diamétrica estima o número de árvores e a produção por hectare por classe de diâmetro, e a produção total pode ser obtida por meio do somatório da variável de interesse em todas as classes de diâmetro (CAMPOS; LEITE, 2013). Esses modelos se baseiam em funções densidade de probabilidade (f.d.p.) que estimam a frequência de árvores nas diferentes classes de diâmetros e possibilitam a avaliação do crescimento da floresta visando produzir multiprodutos da madeira, visto que diferenciam a produção em relação à classe de tamanho das árvores (SIIPILEHTO et al., 2007).

Dentre as funções densidade de probabilidade, a função Weibull destaca-se por sua relativa simplicidade de ajuste e flexibilidade para representar diferentes comportamentos de distribuição diamétrica (BAILEY; DELL, 1973). Essa função ainda tem a vantagem de seus parâmetros serem facilmente correlacionados com variáveis do povoamento (CAMPOS; LEITE, 2013); tem sido amplamente utilizada em estudos que envolvem distribuição diamétrica no Brasil, principalmente em povoamentos de eucalipto, pinus e teca (NOGUEIRA et al., 2006; SOARES et al., 2011; STEPKA et al., 2011; TÉO et al., 2011).

As equações de regressão utilizadas para estimar os parâmetros da função densidade de probabilidade comumente empregam variáveis independentes características do povoamento, como idade, número de árvores por hectare e índice de local (CASTRO et al., 2016; LEITE et al., 2005; NOGUEIRA et al., 2005), a área basal, no entanto, não tem sido utilizada nos sistemas de equações para florestas no Brasil. Essa variável constitui um importante parâmetro da densidade do povoamento, fornecendo o grau de ocupação de determinada área por árvores (CAMPOS; LEITE, 2013), além disso, pode indicar a estagnação do crescimento florestal. É um índice de densidade utilizado para tomar decisões de manejo, fundamentalmente nas decisões de oportunidade e intensidade dos desbastes (CRECHI, 1996).

Assim, o objetivo do presente trabalho foi ajustar um modelo em nível de distribuição diamétrica com inclusão da variável área basal no sistema de equações utilizado para projetar os parâmetros da função Weibull e avaliar a eficiência desse modelo na projeção da distribuição diamétrica de plantios comerciais de Eucalyptus.

\section{MATERIAL E MÉTODOS}

Os dados utilizados nesse estudo foram obtidos de inventários florestais contínuos de clones do híbrido de Eucalyptus urophylla x Eucalyptus grandis não desbastados. O povoamento possui 4.052 hectares e está inserido na região Noroeste do Estado de Minas Gerais. O clima da região é o tropical úmido de savana, com inverno seco e verão chuvoso, do tipo Aw, conforme a classificação de Köppen. A temperatura média anual é de $22,6^{\circ} \mathrm{C}$, tendo uma média mensal de $18^{\circ} \mathrm{C}$ na estação mais fria e $29,1^{\circ} \mathrm{C}$ na mais quente e precipitação média anual é de $1.450 \mathrm{~mm}$.

A modelagem do crescimento e produção em nível de distribuição diamétrica foi realizada com a inclusão da área basal como variável explanatória de densidade, a partir de 264 parcelas permanentes com formato retangular, sendo sua área média $291,5 \mathrm{~m}^{2}$, as quais foram mensuradas em idades variando de 19 a 98 meses, cada parcela medida no mínimo em três ocasiões. Em cada ocasião foram mensurados o diâmetro dos indivíduos a 1,30m de altura $(D A P, \mathrm{em} \mathrm{cm})$, considerando o nível de inclusão de 4,0cm, com auxílio de uma suta; e a altura total das 10 primeiras árvores de cada parcela utilizando o clinômetro digital.

A sequência para ajuste do modelo de distribuição diamétrica foi a seguinte: a) obtenção de dados de parcelas permanentes, de maneira representativa; b) ajuste de uma função densidade de probabilidade para cada parcela, em cada medição; c) obtenção (seleção) de equações de regressão para estimar os parâmetros da $f d p$ na idade futura, utilizando como variáveis independentes características do povoamento, incluindo a área basal; e d) avaliação e validação (aplicação) da equação, conforme sugerido por Campos e Leite (2013).

As árvores, de cada parcela e em cada idade, foram agrupadas em classes com amplitude de $2,0 \mathrm{~cm}$ de diâmetro, conforme sugerido por Araújo Júnior et al. (2010), sendo o limite inferior da primeira classe definido com base no diâmetro mínimo. Para descrever a distribuição diamétrica de cada 
parcela em cada idade, foi ajustada a função densidade de probabilidade Weibull de dois parâmetros (Eq. 1) pelo método da máxima verossimilhança, empregando o algoritmo desenvolvido por Gove e Fairwheater (1989), por meio do software Weibull Trunc (PELLI, ARAÚJO JÚNIOR e MIRANDA, 2007).

$$
f(X)=\frac{\gamma}{\beta}\left(\frac{X}{\beta}\right)^{\gamma-1} e^{-\left(\frac{X}{\beta}\right)^{\gamma}}
$$

Em que: $f(X)=$ frequência de árvores na classe de DAP $X ; X=$ centro de classe de diâmetro, em cm; $\beta=$ parâmetro de escala; $\gamma=$ parâmetro de forma.

A qualidade dos ajustes foi avaliada pelo teste de Kolmogorov-Smirnov $(\alpha=0,01)$ a fim de verificar a aderência da função Weibull aos dados. Esse teste consiste no cálculo da máxima diferença entre a frequência acumulada observada e a frequência acumulada estimada, sendo a classe de maior divergência a estatística do teste $(d n)$ (Eq. 2) e, em seguida, esse valor é comparado com o tabelado ao nível de 1\% de significância (SOKAL; ROHLF, 1981). Neste teste quanto maior o valor de $d n$, maior é a divergência entre os valores reais e os valores estimados pela função.

$$
d n=M A X|F(X)-S(X)|
$$

Em que: $S(X)$ é a frequência acumulada observada e $F(X)$ é a frequência estimada pela função Weibull.

A redistribuição teórica dos diâmetros por classe ou recuperação da distribuição diamétrica foi realizada por meio da alternativa tradicional de correlacionar parâmetros da função com características do povoamento. Essa correlação foi feita por meio de regressão linear, sendo as variáveis dependentes os parâmetros da função Weibull em uma idade futura e, como variáveis independentes, os parâmetros dessa mesma função em uma idade atual e as características do povoamento em idades atual e futura.

Os seguintes atributos do povoamento foram utilizados: idade, diâmetro máximo, diâmetro mínimo, diâmetro médio, número de árvores, área basal e combinações dessas variáveis. O sistema de equações utilizado para projetar os parâmetros da $f d p$ foi baseado nos sistemas propostos por Binoti et al. (2010) e Nogueira (2003), com adaptações e inclusão da variável área basal. Foram ajustadas cinco equações para cada variável, sendo a melhor selecionada para compor o sistema de equações (Eq. 3 a 9). Para ajustar as equações utilizou-se o Método dos Mínimos Quadrados Ordinários (GUJARATI; PORTER, 2011). O sistema de equações selecionado foi:

$$
\begin{aligned}
& B_{2}=c_{0}+c_{1}\left(\frac{I_{1}}{I_{2}}\right) B_{1}+c_{2}\left(1-\frac{I_{1}}{I_{2}}\right) d \max _{1}+\varepsilon \\
& d \max _{2}=c_{0}+c_{1}\left(\frac{I_{1}}{I_{2}}\right) d \max _{1}+c_{2}\left(1-\frac{I_{1}}{I_{2}}\right) B_{1}+c_{3}\left(1-\frac{I_{1}}{I_{2}}\right) q_{1}+\varepsilon \\
& d \min _{2}=c_{0}+c_{1} d \min _{1}+c_{2} B_{1}+c_{3} I_{1}^{-1}+\varepsilon \\
& q_{2}=c_{0}+c_{1} q_{1}+c_{2}\left(1-\frac{I_{1}}{I_{2}}\right) d \max _{1}+c_{3}\left(\frac{I_{1}}{I_{2}}\right) B_{1}+\varepsilon \\
& N_{2}=c_{0}+c_{1} \cdot N_{1}+c_{2} \cdot\left(\frac{I_{1}}{I_{2}}\right) B_{I}+c_{3}\left(1-\frac{I_{I}}{I_{2}}\right)\left(d \max _{1}-q_{1}\right)+\varepsilon \\
& \beta_{2}=c_{1} \beta_{1}+c_{2}\left(1-\frac{I_{1}}{I_{2}}\right)\left(d \max _{1}-q_{1}\right)+c_{3}\left(\frac{I_{1}}{I_{2}}\right) B_{I}+\varepsilon \\
& \gamma_{2}=c_{1} \gamma_{1}+c_{2}\left(1-\frac{I_{1}}{I_{2}}\right) d \max _{1}+c_{3}\left(\frac{I_{1}}{I_{2}}\right) B_{1}+\varepsilon
\end{aligned}
$$

Em que: $I_{1}$ e $I_{2}=$ idades atual e futura, em meses; $B_{1}$ e $B_{2}=$ área basal nas idades atual (1) e futura (2), $\mathrm{em} \mathrm{m}^{2} \cdot \mathrm{ha}^{-1} ; d \max _{1} \mathrm{e} d \max _{2}=$ diâmetros máximos nas idades atual (1) e futura (2), em cm; $d \min _{1}$ e $d \min _{2}=$ diâmetros mínimos nas idades atual (1) e futura (2), em cm; $\mathrm{q}_{1}$ e $\mathrm{q}_{2}=$ diâmetro médio nas idades atual (1) e futura (2); $N_{1}$ e $N_{2}$ = frequências de árvores por hectare nas idades atual (1) e futura (2); $\beta_{1}$ e $\beta_{2}$ = parâmetros de escala da função Weibull nas idades atual (1) e futura (2); $\gamma_{1}$ e 
$\gamma_{2}=$ parâmetros de forma da função Weibull nas idades atual (1) e futura (2); $c_{\mathrm{i}}=$ parâmetros dos modelos e $\varepsilon=$ erro aleatório.

Para identificar possíveis inconsistências nas estimativas das variáveis, além da distribuição gráfica dos resíduos, foram calculadas as estatísticas de coeficiente de determinação ajustado $\left(R_{\text {aj. }}^{2}\right)$ (Eq. 10) e erro padrão da média relativo $\left(\mathrm{S}_{\mathrm{y}, \mathrm{x} \%}\right)$ (Eq. 11), definidos por:

$$
\begin{gathered}
R_{a j .}^{2}=1-\left(\frac{n-1}{n-p-1}\right)\left(1-\frac{\sum_{i=1}^{n}\left(y_{i}-\hat{y}_{i}\right)^{2}}{\sum_{i=1}^{n}\left(y_{i}-\bar{y}\right)^{2}}\right) \\
S_{y . x \%}=\frac{\sqrt{\frac{\sum\left(y_{i}-\hat{y}_{i}\right)^{2}}{n-p-1}}}{\bar{y}} \times 100
\end{gathered}
$$

Em que: $n=$ número de observações; $\gamma_{i}=$ valores observados para as variáveis; $\hat{y}_{i}=$ valores estimados para as variáveis na i-ésima observação; $\bar{y}=$ valor médio observado para as variáveis; $\mathrm{n}$-p-1 = grau de liberdade dos resíduos da equação.

O ajuste do sistema de equações e da função Weibull foi realizado utilizando dados de $80 \%$ das parcelas e a validação realizada com os $20 \%$ de dados restantes, conforme realizado por Castro et al. (2016). A distribuição diamétrica foi projetada a partir dos dados da medição realizada em uma primeira ocasião para a idade seguinte e assim sucessivamente até a última idade de cada parcela. Em seguida os valores estimados foram comparados com os valores observados por meio do teste de Kolmogorov-Smirnov $(\alpha=0,01)$.

\section{RESULTADOS E DISCUSSÃO}

O ajuste da função densidade de probabilidade Weibull de dois parâmetros foi realizado para cada parcela em cada idade, totalizando 1014 ajustes, sendo aderente em $98 \%$ dos casos pelo teste de Kolmogorov-Smirnov ao nível de 1\% de significância.

As equações ajustadas também resultaram em coeficientes de determinação ajustados $\left(R_{\text {aj }}^{2}\right.$ ) altos, com valores maiores que 0,90 . O erro padrão relativo da média $\left(\mathrm{S}_{\mathrm{y}, \mathrm{x} \%}\right)$ para cada uma das equações foi baixo, menores que $15 \%$, podendo inferir que houve baixa variação entre dados estimados e observados (Tabela 1). Essa relação estreita é fundamental, pois os parâmetros estimados serão aplicados para obtenção da distribuição futura de forma eficiente (precisa).

As equações selecionadas para as variáveis de povoamento, com inclusão da área basal, apresentaram alta qualidade de ajuste, com dispersão de resíduos baixa e homocedástica. Já aquelas equações selecionadas para estimar os parâmetros da função Weibul 2P apresentaram dispersão de resíduos maior do que aquela observada para as variáveis de povoamento, apresentando tendência de variância menos constante dos erros, entretanto todas as variáveis apresentaram tendência à distribuição normal dos resíduos (Figura 1).

Tabela 1. Coeficientes das equações ajustadas para as variáveis do povoamento: área basal $\left(\mathrm{B}_{2}\right), D A P$ máximo $\left(\mathrm{d}_{\max 2}\right)$, $D A P$ mínimo $\left(\mathrm{d}_{\min 2}\right)$, diâmetro médio $\left(\mathrm{q}_{2}\right)$ e frequência $\left(\mathrm{N}_{2}\right.$, árvores.ha $\left.{ }^{-1}\right)$; e parâmetros da função Weibull: beta $\left(\beta_{2}\right)$ e gama $\left(\gamma_{2}\right)$ em idade futura e suas respectivas estatísticas de coeficiente de determinação ajustado $\left(\mathrm{R}^{2}{ }_{\text {aj. }}\right.$ ) e erro padrão da média relativo $\left(\mathrm{S}_{\mathrm{y}, \mathrm{x}} \%\right)$.

Table 1. Coefficients of the equations adjusted for the variables of the stand: basal area $\left(B_{2}\right)$, maximum DAP $\left(\mathrm{d}_{\max 2}\right)$, DAP minimum $\left(\mathrm{d}_{\min 2}\right)$, mean diameter $\left(\mathrm{q}_{2}\right)$ and frequency $\left(\mathrm{N}_{2}\right.$, trees.ha-1); and parameters of the Weibull function: beta $\left(\beta_{2}\right)$ and gamma $\left(\gamma_{2}\right)$ at future age and their respective coefficient of determination statistics adjusted $\left(\mathrm{R}_{\mathrm{aj} .}^{2}\right)$ and standard error of the relative mean $\left(\mathrm{S}_{\mathrm{y}, \mathrm{x}} \%\right)$.

\begin{tabular}{ccccccc}
\hline Variáveis & $\mathrm{C}_{0}$ & $\mathrm{C}_{1}$ & $\mathrm{C}_{2}$ & $\mathrm{C}_{3}$ & $\mathbf{R}_{\mathrm{Aj} .}$ & $\mathrm{S}_{\mathrm{y}, \mathrm{x}}(\%)$ \\
\hline $\mathrm{B}_{2}$ & 9,0615 & 1,2091 & $-0,8196$ & - & 0,95 & 4,80 \\
$\mathrm{~d}_{\max 2}$ & 1,9408 & 0,8928 & $-0,5277$ & 2,2248 & 0,92 & 3,48 \\
$\mathrm{~d}_{\min 2}$ & $-0,8179$ & 1,0355 & 15,6743 & $-0,0293$ & 0,94 & 6,24 \\
$\mathrm{q}_{2}$ & $-3,9843$ & 1,1511 & 68,6400 & $-0,0532$ & 0,93 & 3,03 \\
$\mathrm{~N}_{2}$ & 0,9338 & 1,0009 & 0,0542 & $-1,6134$ & 0,99 & 0,87 \\
$\boldsymbol{\beta}_{2}$ & - & 0,9108 & 1,4189 & 0,2335 & 0,91 & 11,23 \\
$\mathrm{Y}_{2}$ & - & 0,8677 & 0,0990 & $-0,0648$ & 0,90 & 14,73 \\
\hline
\end{tabular}



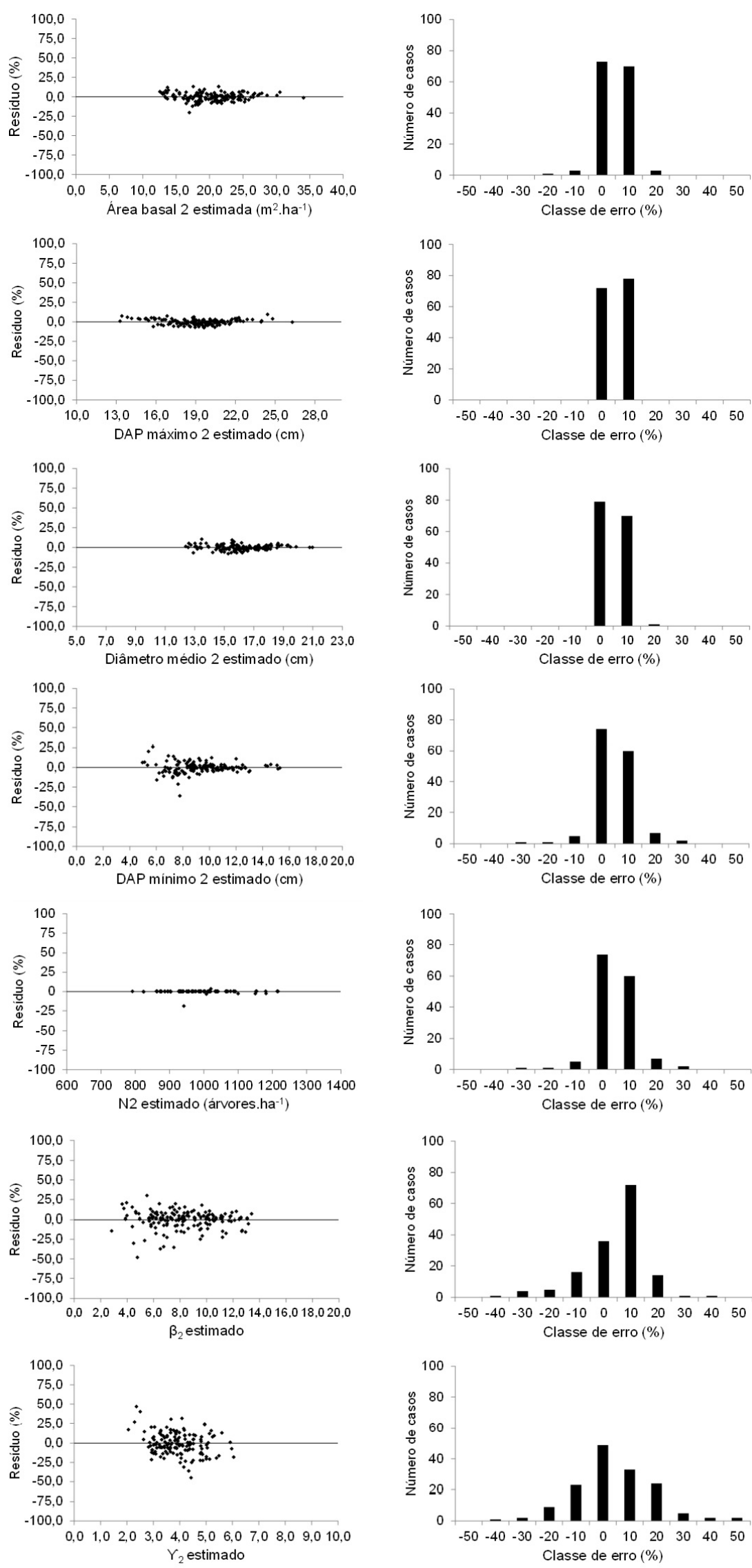

Figura 1. Gráficos de dispersão e histogramas da distribuição de resíduos para estimativas da área basal, diâmetro máximo, diâmetro mínimo, diâmetro médio, frequência de árvores por hectare e os parâmetros Beta $(\beta)$ e Gama ( $(\mathrm{\gamma})$ da função Weibul em idade futura de plantio comercial do híbrido de Eucalyptus urophylla x Eucalyptus grandis.

Figure 1. Dispersion graphic and histograms of the distribution of residuals for estimates of basal area, maximum diameter, minimum diameter, mean diameter, tree frequency per hectare and the Beta $(\beta)$ and Range (y) parameters of the Weibul function at future commercial planting age of the Eucalyptus urophylla $x$ Eucalyptus grandis hybrid. 
A utilização da função Weibull tem sido estudada em diversos trabalhos na área florestal, e as características já mencionadas das estimativas dos parâmetros dessa função têm sido observadas, embora esses estudos não tenham utilizado a área basal na projeção desses parâmetros (CASTRO et al., 2016; LEITE et al., 2013; SOARES et al., 2010). Para povoamentos de região de clima temperado, no entanto, observou-se a existência de uma estreita relação entre os parâmetros da função Weibull, especialmente Beta, e a área basal (HÖKKÄ et al., 1991; MALTAMO et al., 1995).

Para representar a prognose da distribuição diamétrica do povoamento selecionou-se uma parcela ao acaso. A tendência de crescimento representada apresentou comportamento coerente com a teoria, de acordo com a qual as distribuições se achatam e deslocam para a direita. Assim tem-se que a distribuição diamétrica estimada é consistente do ponto de vista do realismo biológico (Figura 2)
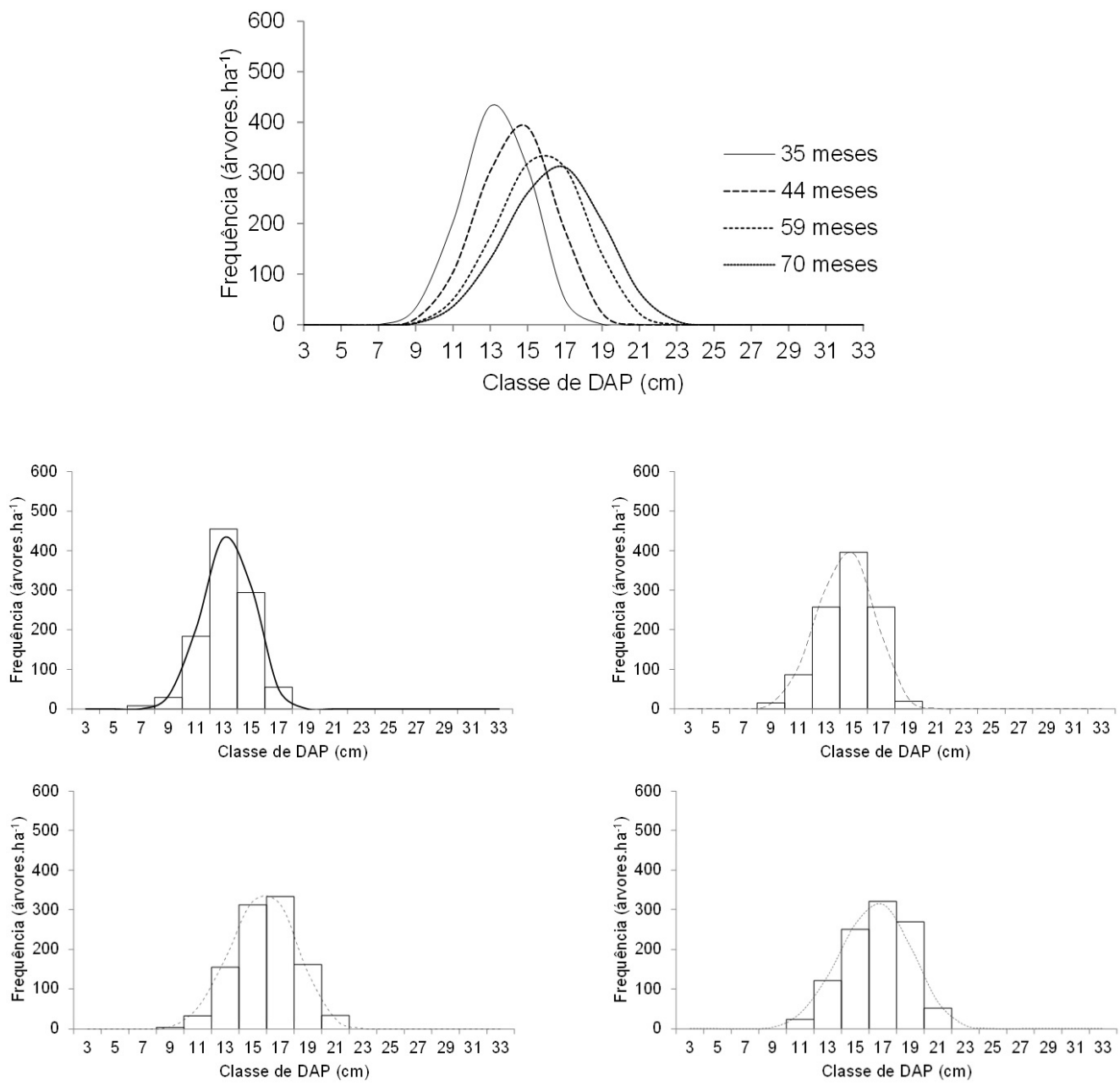

Figura 2. Dstribuição diamétrica estimada em quatro prognoses para a parcela selecionada; e comparação entre distribuição diamétrica observada (barras) e estimada (linhas) em cada uma das prognoses de crescimento de plantio comercial do híbrido de Eucalyptus urophylla x Eucalyptus grandis.

Figure 2. Diametric distribution estimated in four prognoses for the selected plot; and comparison between observed (bars) and estimated (row) diametric distribution in each of the growth prognoses of commercial planting of Eucalyptus urophylla x Eucalyptus grandis.

As comparações entre distribuições diamétricas observadas e estimadas em cada idade permitem inferir que o sistema de equações de projeção do crescimento proporcionaram estimativas precisas. Além disso, essas observações confirmam que a quantificação da distribuição diamétrica e sua relação, entre outras, com variáveis explanatórias de densidade, como é caso de área basal, é importante tanto econômico como biologicamente (BAILEY; DELL, 1973), pois a distribuição diamétrica é um indicador do estoque de crescimento da floresta (LOETSCH et al., 1973). 
Na comparação entre prognoses com e sem utilização da área basal no sistema de equações observa-se a importância dessa variável para as estimativas obtidas. Em ambos os casos a primeira prognose foi obtida com alta precisão, observando redução desta, em diferentes proporções, nas idades seguintes.

Para prognoses com utilização da área basal, a maior perda de precisão entre prognoses sucessivas foi de 5,5\%, ocorrendo da terceira para a quarta e, uma redução total de 10,4\% entre primeira para a última prognose (Figura 3a). Já prognose sem utilização da área basal apresentou perda de precisão de até 9,3\% entre prognoses sucessivas e de 22,3\% entre a primeira e a última prognose (Figura 3b). As diferenças entre as prognoses com e sem área basal no sistema de equações, em cada idade, aumentaram progressivamente, sendo de 4,$8 ; 10,2 ; 15,0$ e $17,4 \%$ entre a $1^{\mathrm{a}}, 2^{\mathrm{a}}, 3^{\mathrm{a}}$ e $4^{\mathrm{a}}$ prognoses, respectivamente.

(a)

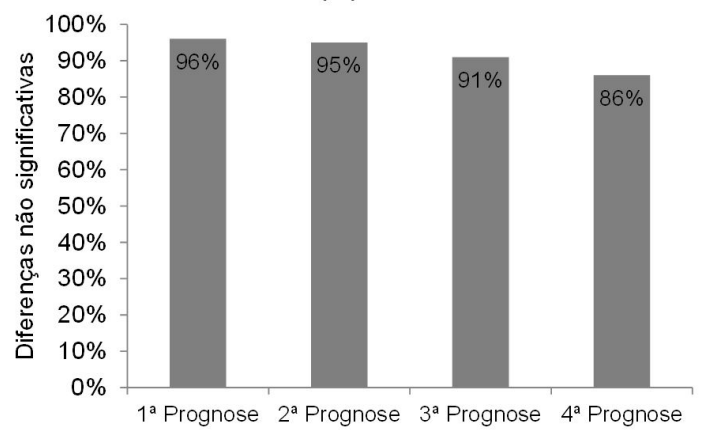

(b)

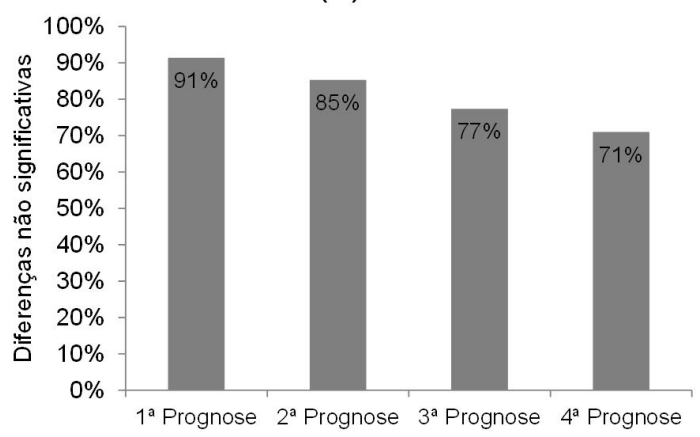

Figura 3. Porcentagem de parcelas nas quais os valores estimados do número de árvores por classe diamétrica em prognoses, com (a) e sem (b) a utilização da área basal no sistema de equações, não diferiram significativamente dos valores observados para plantio comercial do híbrido de Eucalyptus urophylla x Eucalyptus grandis.

Figure 3. Percentage of plots in which the estimated values of the number of trees per diameter class in prognoses with (a) and without (b) the use of the basal area in the equation system did not differ significantly from the values observed for commercial planting of the Eucalyptus hybrid urophylla $x$ Eucalyptus grandis.

A modelagem da distribuição diamétrica utilizando as variáveis do povoamento, como diâmetro máximo, diâmetro mínimo, diâmetro médio e densidade, em idades atual e futura resultando em um sistema de projeção já haviam sido propostos por Nogueira (2003), e posteriormente foram utilizados por Binoti et al. (2010) e Leite et al. (2005), sendo comprovado eficiência dos mesmos. Os sistemas aqui utilizados incluem, além das variáveis de povoamento previamente mencionadas, a área basal e também foram eficientes. Considerando que os plantios florestais constituem sistemas biológicos dinâmicos que passam por constantes mudanças, essa variável, que também é influenciada pelo crescimento diamétrico e densidade de indivíduos, contribui para a projeção dessas mudanças, sendo importante suporte à tomada de decisões sobre intervenções no povoamento florestal.

\section{CONCLUSÃO}

A distribuição diamétrica de povoamento comercial de eucalipto projetada com a inclusão de área basal no sistema de equações para projeção dos parâmetros da função Weibull de dois parâmetros apresenta consistência, além de ser eficiente para, pelo menos, quatro prognoses consecutivas.

\section{REFERÊNCIAS}

ARAÚJO JÚNIOR, C. A.; NOGUEIRA, G. S.; OLIVEIRA, M. L. R.; MIRANDA, R. O. V.; CASTRO, R. V. O.; PELLI, E. Projeção da distribuição diamétrica de povoamentos de Eucalyptus em diferentes amplitudes de classe. Pesquisa Agropecuária Brasileira, Brasília, v. 45, n. 11, p. 1275-1281, 2010.

BAILEY, R. L.; DELL, T. R. Quantifying diameter distributions with the Weibull function. Forest Science, v.19, n.2, p.97-104, 1973.

BINOTI, D. H. B.; LEITE, H. G.; NOGUEIRA, G. S.; SILVA, M. L. M.; GARCIA, S. L. R.; CRUZ, J. P. Uso da função Weibull de três parâmetros em um modelo de distribuição diamétrica para plantios de eucalipto submetidos a desbaste. Revista Árvore, Viçosa, v. 34, n. 1, p. 147-156, 2010. 
Costa et al. - Projeção da distribuição de povoamento comercial de eucalipto

utilizando área basal

BURKHART, H.E.; TOMÉ, M. Modeling forests trees and stands. New York: Springer, 2012. 459 p.

CAMPOS, J. C. C.; LEITE, H. G. Mensuração florestal: perguntas e respostas. 4. ed. Viçosa, MG: UFV, 2013.605 p.

CASTRO, R. V. O.; ARAúJO, R. A. A.; LEITE, H. G.; CASTRO, A. F. N. M.; SILVA, A.; PEREIRA, R. S.; LEAL, F. A. Modelagem do crescimento e da produção de povoamentos de Eucalyptus em nível de distribuição diamétrica utilizando índice de local. Revista Árvore, Viçosa, v. 40, n. 1, p. 107-116, 2016.

CRECHI, E.H. Efeitos da densidade da plantação sobre a produção, crescimento e sobrevivência de Araucaria angustifolia (Bert.) O. Ktze. em Missiones, Argentina. 1996. 172 p. Dissertação (Mestrado em Ciências Florestais) - Universidade Federal do Paraná, Curitiba, 1996.

GOVE, J. H.; FAIRWEATHER, S. E. Maximum likelihood estimation of Weibull function parameters using a general interactive optimizer and grouped data. Forest Ecology and Management, Amsterdan, v. 28, n. 1, p. 61-99, 1989.

GUJARATI, D. N.; PORTER, D. C. Econometria básica. 5. ed. Porto Alegre: AMGH, 2011. 924 p.

HÖKKÄ, H.; PIIROINEN, M.L.; PENTTILÄ, T. The estimation of basal area - dbh distribution using the Weibull function for drained pine and birch dominated and mixed peatland stands in north Finland. Folia Forestalia, v. 781, p. 1-22, 1991.

LEITE, H. G.; ALCÂNTARA, A. E. M.; BINOTI, D. H. B.; OLIVEIRA NETO, R. R.; SILVA, M. L. M. Comparação entre predição e projeção da distribuição de diâmetros de povoamentos de eucalipto submetidos a desbastes. Revista Árvore, Viçosa, v. 37, n. 2, p. 321-328, 2013.

LEITE, H. G.; NOGUEIRA, G. S.; CAMPOS, J. C. C.; SOUZA, A. L.; CARVALHO, A. Avaliação de um modelo de distribuição diamétrica ajustado para povoamentos de Eucalyptus sp. submetidos a desbaste. Revista Árvore, Viçosa, v. 29, n. 2, p. 271-280, 2005.

LOETSCH, F.; ZOHRER, F.; HALLER, R.E. Forest inventory. München: BLV Verlagsgesellschaft, 1973. v. 2, 472 p.

MALTAMO, M.; PUUMALAINEN, J.; PAIVINEN, R. Comparison of beta and Weibull functions for modeling basal area diameter distribution in stands of Pinus sylvestris and Picea abies. Scandinavian Journal Forest Research, v. 10, p. 284-295, 1995.

NOGUEIRA, G.S. Modelagem do crescimento e da produção de povoamentos de Eucalyptus sp.e de Tectona grandis submetidos a desbaste. 2003. 132 p. Tese (Doutorado em Ciência Florestal) - Universidade Federal de Viçosa, Viçosa, 2003.

NOGUEIRA, G. S.; LEITE, H. G.; CAMPOS, J. C. C.; TAKIZAWA, F. H.; COUTO, L. Avaliação de um modelo de distribuição diamétrica ajustado para povoamentos de Tectona grandis submetidos a desbaste. Revista Árvore, v. 30, n. 3, p. 377-387, 2006

NOGUEIRA, G. S.; LEITE, H. G.; CAMPOS, J. C. C.; CARVALHO, F. A.; SOUZA, A. L. Modelo de distribuição diamétrica para povoamentos de Eucalyptus sp. submetidos a desbaste. Revista Árvore, Viçosa, v.29, n.4, p.579-589, 2005.

PELLI, E.; ARAÚJO JÚNIOR, C. A.; MIRANDA, R. O. V. Weibull Trunc (software não registrado).

SIIPILEHTO, J.; SARKKOLA, S.; MEHTÄTALO, L. Comparing regression estimation techniques when predicting diameter distributions of Scots pine on drained peatlands. Silva Fennica, Helsinski, v. 41, n. 2, p. 333-349, 2007.

SOARES, T. S.; LEITE, H. G.; SOARES, C. P. B.; VALE, A. B. Projeção da distribuição diamétrica e produção de povoamentos de eucalipto empregando diferentes formas da função Weibull. Revista Árvore, Viçosa, v. 35, n. 5, p. 1027-1032, 2011.

SOARES, T. S.; LEITE, H. G.; SOARES, C. P. B.; VALE, A. B. Procedures for theoretical redistribution of diameters in eucalyptus stands. Cerne, Lavras, v. 16, n. 1, p. 68-76, 2010.

SOKAL, R. R.; ROHLF, F.J. Biometry: the principles and practice of statistics in biological research. San Francisco: Freeman, $1969.776 \mathrm{p}$.

STEPKA, T. F.; LISBOA, G. S.; KURCHAIDT, S. M. Funções densidade de probabilidade para a estimativa da distribuição diamétrica em povoamento de Eucalyptus sp. na região centro-sul do Paraná. Ambiência, v. 7, n. 3, p. 429-439, 2011.

TÉO, S. J.; ROCHA, S. P.; BORTONCELLO, A. C.; PAZ, R. A.; COSTA, R. H. Dinâmica da distribuição diamétrica de povoamentos de Pinus taeda, na região de Caçador, SC. Pesquisa Florestal Brasileira, Colombo, v. 31, n. 67, p. 183-192, 2011.

Recebido em: 16/03/2018

Aceito em: 24/09/2018

Sci. For., Piracicaba, v. 47, n. 122, p. 265-272, jun. 2019

DOI: doi.org/10.18671/scifor.v47n122.09 